\title{
POLYNOMIAL CHARACTERIZATION OF TWIN PRIMES IN FUNCTION OF ANOTHER PRIME
}

\author{
Ibrahima Gueye* \\ *Mathematician amator
}

Keywords: Additive primes numbers theory; twin primes;

\begin{abstract}
For two millennia, the prime numbers have continued to fascinate mathematicians. Indeed, a conjecture which dates back to this period states that the number of twin primes is infinite. In 1949 Clement showed a theorem on twin primes. In this paper I give the proof of a polynomial characterization of twin primes using additive primes number theory.
\end{abstract}

\section{Introduction:}

Or $(p, q)$ a pair of integers such that $\mathrm{p}$ and $\mathrm{q}$ are both prime and $\mathrm{p}<\mathrm{q}$. We say that $(\mathrm{p}, \mathrm{q})$ form a pair of twin primes if $q=p+2$.

The couple $(2,3)$ is the only pair of consecutive primes.

Omitting the pair $(2,3), 2$ is the smallest possible distance between two primes, twin primes are two and two consecutive odd numbers.

Any pair of twin primes (with the exception of the couple $(3,5))$ is of the form $(6 n-1,6 n+1)$ for some integer $\mathrm{n}$. Indeed, any set of three consecutive natural numbers has at least a multiple of 2 (possibly two) and one multiple of 3, these two are confused between multiple two twin primes.

It is possible to show that, for any integer, the pair $(m, m+2)$ consists of twin primes if and only if:

$$
4[(\mathrm{~m}-1) !+1]+\mathrm{m}=0 \quad \bmod \mathrm{m}(\mathrm{m}+2)
$$

This characterization of factorial and modular twin primes was discovered by P. A. Clement in 1949[2].

The series of reciprocals of twin primes converges to Brun's constant, unlike the series of reciprocals of prime numbers. This property was demonstrated by Viggo Brun in 1919 [4].

The twin prime conjecture states that there are infinitely many twin primes. In other words, there are infinitely many primes $\mathrm{p}$ such that $(\mathrm{p}+2)$ is also prime.

In 1940, Paul Erdős proved the existence of a constant $\mathrm{c}<1$ and infinitely many primes $\mathrm{p}$ such that: $p^{\prime}-p<\operatorname{cln}(p)$ where $p^{\prime}$ denotes the number immediately following the first $p$.

This result was improved several times, in 1986, Helmut Maier showed a constant $\mathrm{c}<0.25$ could be reached.

In 2003, Daniel Goldston and Cem Yildirim have shown that, for all $c>0$, there are infinitely many primes $p$ such that $\mathrm{p}^{\prime}-\mathrm{p}<\mathrm{cln}(\mathrm{p})$.

In 1966, Chen Jingrun demonstrated the existence of infinitely many primes $p$ such that $p+2$ is the product of at most two prime factors (such a number, product of at most two prime factors, 2 is said -almost-first).

His approach was that of the theory of the screen, he used to treat similarly the twin prime conjecture and Goldbach's Conjecture.

Between 2006 and 2009, Ben Green, Terence Tao and Tamar Ziegler gave in several of their articles that there is an infinity of integers $n$ and $m$ tells that: $n, m,(n+m+1)$ and $(n+2 m+2)$ are prime-numbers[3].

In this paper i proove a polynomial characterization of twin primes using additive primes number theory. 


\section{Theorem 1: Theorem of Wilson}

Statement: An integer $\mathrm{p}$ strictly greater than 1 , is a prime number if and only if divides $(\mathrm{p}-1) !+1$, that is to say if and only if:

$$
(p-1) !+1=0(\bmod p)
$$

\section{Theorem 2: Theorem of Clement}

Statement: For any integer, the pair $(\mathrm{m}, \mathrm{m}+2)$ consists of twin primes if and only if $4[(m-1) !+1]+m=0 \quad \bmod m(m+2)$

\section{Theorem 3:}

Statement: There are infinitely many integers $n$ and $m$ tells that: $n, m,(n+m+1)$ and $(n+2 m+2)$ are prime numbers [3].

\section{Theorem 4:}

For a positive odd integer $\mathrm{p}$ and for any two distinct odd primes $\mathrm{p} 1$ and $\mathrm{p} 2$ with $\mathrm{p} 1+\mathrm{p} 2-\mathrm{p}=1$, then $(p-p 1) !(p-p 2) !=-1(\bmod p) \Leftrightarrow p$ is prime[1].

\section{Theorem 5:}

Given primes numbers $\mathrm{m},(\mathrm{m}+2)$ and $(\mathrm{n}+2)=>\mathrm{m}$ and $(\mathrm{m}+2)$ are twin primes.

We can find integers $\mathrm{c}$ and $\mathrm{d}$ so that:

$$
d^{*} m^{2 *} m+(2 * d+d * n-1) * m^{2}+(d * n+d-c-1) * m-c * n-3 * c+1=0
$$

We have a particular case if $\mathrm{n}=5$. In this case: $\{m ;(m+2) ;(m+6) ;(m+8)\}$ is quadruple of primes.

$$
d^{*} m^{2 *} m+(7 * d-1)^{*} m^{2}+(6 * d-c-1)^{*} m-8 * c+1=0
$$

\section{Proof:}

Given primes numbers $m,(m+2)$ and $(n+2)$

According to Theorem 4:

$((\mathrm{n}+\mathrm{m}+3)-(\mathrm{n}+2)) ! *((\mathrm{n}+\mathrm{m}+3)-(\mathrm{m}+2)) !=(-1) \operatorname{modulo}(\mathrm{n}+\mathrm{m}+3)$

According to the same Theorem 4 :

$$
\begin{aligned}
& ((\mathrm{n}+\mathrm{m}+1)-(\mathrm{n}+2)) ! *((\mathrm{n}+\mathrm{m}+1)-\mathrm{m}) !=(-1) \operatorname{modulo}(\mathrm{n}+\mathrm{m}+1) \\
& \Rightarrow(\mathrm{n}+1) !=(\mathrm{c} *(\mathrm{n}+\mathrm{m}+3)-1) /(\mathrm{m}+1) !=(\mathrm{d} *(\mathrm{n}+\mathrm{m}+1)-1) /(\mathrm{m}-1) ! \\
& \Rightarrow(\mathrm{c} *(\mathrm{n}+\mathrm{m}+3)-1) /(\mathrm{m} *(\mathrm{~m}+1))=(\mathrm{d} *(\mathrm{n}+\mathrm{m}+1)-1) \\
& \mathrm{d} * \mathrm{~m}^{2 *} \mathrm{~m}+(2 * \mathrm{~d}+\mathrm{d} * \mathrm{n}-1) * \mathrm{~m}^{2}+(\mathrm{d} * \mathrm{n}+\mathrm{d}-\mathrm{c}-1) * \mathrm{~m}-\mathrm{c} * \mathrm{n}-3 * \mathrm{c}+1=0
\end{aligned}
$$

For $m,(m+2)$ and $(n+2)$ prime numbers, if we proove that this equation have infinity solutions we can conclude of the infinity of twin primes. 
For $\mathrm{n}=5$ :

$\mathrm{n}+\mathrm{m}+1=\mathrm{m}+6$ et $\mathrm{n}+\mathrm{m}+3=\mathrm{m}+8$

$\{\mathrm{m} ;(\mathrm{m}+2) ;(\mathrm{m}+6) ;(\mathrm{m}+8)\}$ is quadruple of primes.

The equation begin:

$d^{*} m^{2 *} m+(7 * d-1)^{*} m^{2}+(6 * d-c-1)^{*} m-8 * c+1=0$

\section{References:}

[1] K. Raja Rama Gandhi, Super six problems on primes, International Journal of Advancements in Research \& Technology, Volume 2, Issue1, January-2013

[2] PA Clement, Congruences for sets of premiums, American Mathematical Monthly 56 (1949), p. 23-25

[3] Terence Tao, University of California, Los AngelesMahler Lecture Series

[4] Viggo Brun, Series $1 / 5+1 / 7+1 / 11+1 / 13+1 / 17+1 / 19+1 / 29+1 / 31+1 / 41+1 / 43+1 / 59+$ $1 / 61+\ldots$ where denominators are "twin primes" is convergent or over, Bulletin of Mathematical Sciences 43 (1919), p. 100-104 and 124-128 